\title{
The Predictive Ability of Hepatic Steatosis Index for Gestational Diabetes Mellitus and Large for Gestational Age Infant Compared with Other Noninvasive Indices Among Chinese Pregnancies: A Preliminary Double-center Cohort Study
}

\begin{abstract}
Shuoning Song, (1) 'Yanbei Duo,' Yuemei Zhang, ${ }^{2}$ Xiaolin Qiao, ${ }^{3}$ Jiyu $\mathrm{Xu},{ }^{4}$ Jing Zhang, ${ }^{5}$ Zhenyao Peng, Yan Chen, ${ }^{3}$ Xiaorui Nie, ${ }^{3}$ Qiujin Sun, ${ }^{7}$ Xianchun Yang, ${ }^{7}$ Ailing Wang, ${ }^{8}$ Wei Sun, ${ }^{4}$ Yong Fu, ' Yingyue Dong, Zechun Lu, ${ }^{8}$ Tao Yuan, ' Weigang Zhao'
\end{abstract}

'Department of Endocrinology, Key Laboratory of Endocrinology of Ministry of Health, Peking Union Medical College Hospital, Chinese Academy of Medical Science and Peking Union Medical College, Beijing, People's Republic of China;

${ }^{2}$ Department of Obstetrics, Haidian District Maternal and Child Health Care Hospital, Beijing, People's Republic of China; ${ }^{3}$ Department of Obstetrics, Beijing Chaoyang District Maternal and Child Health Care Hospital, Beijing, People's Republic of China; ${ }^{4}$ Core Facility of Instrument, Institute of Basic Medical Sciences, Chinese Academy of Medical Sciences, School of Basic Medicine, Peking Union Medical College, Beijing, People's Republic of China; ${ }^{5}$ Department of Laboratory, Haidian District Maternal and Child Health Care Hospital, Beijing, People's Republic of China; ${ }^{6}$ Department of Dean's Office, Haidian District Maternal and Child Health Care Hospital, Beijing, People's Republic of China; ${ }^{7}$ Department of Clinical Laboratory, Beijing Chaoyang District Maternal and Child Health Care Hospital, Beijing, People's Republic of China; ${ }^{8}$ National Center for Women and Children's Health, China CDC, Beijing, People's Republic of China

Correspondence: Tao Yuan; Weigang Zhao Email t75y@sina.com;

xiehezhaoweigang@163.com
Objective: To evaluate the association of hepatic steatosis index (HSI) in the first trimester and the risk of gestational diabetes mellitus (GDM) as well as large for gestational age (LGA) infant in Chinese women.

Methods: A total of 1082 pregnant women were included in this study. Maternal basic laboratory data, including ALT, AST, FBG, insulin, TG, and HDL-C, were tested during 612 weeks of gestation and anthropometric characteristics were monitored during gestation. A 75-g oral glucose tolerance test (OGTT) was conducted at 24-28 weeks of gestation. HSI, nonalcoholic fatty liver disease (NAFLD) liver fat score, triglyceride/high-density lipoprotein cholesterol (TG/HDL-C) and triglyceride-glucose (TyG) index were calculated. Odds ratio with $95 \%$ confidence interval for subsequent risk of GDM and LGA by HSI quartiles were assessed by binary logistic regression model. The predictive ability of HSI for GDM and LGA was evaluated by the receiver operating characteristic (ROC) curve analysis and was compared with other indices.

Results: The incidence of GDM and LGA were 22.09\% (239/1082) and 10.53\% (87/826). HSI was higher in GDM group than in NGT group (median, interquartile range: 30.67, 27.20-35.10 vs $27.98,25.70-30.82, P<0.001)$. Incidence of GDM was gradually increased with increasing HSI values. Women in the highest HSI quartile had significantly higher risk of LGA delivery than those in the lowest HSI quartile $(P<0.05)$. The area under the ROC curves of HSI for GDM and LGA were higher than other indices, reaching 0.646 (95\% CI: 0.605-0.686) and 0.600 (95\% CI: 0.541-0.660), respectively.

Conclusion: Higher HSI was independently associated with higher risk of GDM and LGA in Chinese women. HSI in the first trimester can predict the risk of GDM and LGA.

Keywords: hepatic steatosis index, nonalcoholic fatty liver disease, gestational diabetes mellitus, large for gestational age infant

\section{Introduction}

Gestational diabetes mellitus (GDM) is one of the most common medical complications of pregnancy, which is described as a state of impaired glucose tolerance first being diagnosed during pregnancy. ${ }^{1}$ It is acknowledged that GDM increases the risk of pregnancy complications and adverse perinatal outcomes, including pregnancy-induced hypertension, preeclampsia, abortion, large for gestational age 
(LGA) delivery and so on, and influenced maternal and infant health in the future. ${ }^{2,3}$ Thus a lot of studies reported risk factors of GDM and aimed to find an effective index to predict GDM.

LGA is defined as birth weight above the 90th percentile for gestational age. LGA increases risks of adverse perinatal outcomes and serious birth complications, such as preterm birth, cesarean section, stillbirth, hypoxic brain damage due to difficulties in delivery, and neonatal hyperinsulinemia. LGA infant have higher risk of developing obesity, type 2 diabetes (T2DM) and other metabolic diseases in the future. ${ }^{4}$ LGA is associated with many risk factors, including maternal BMI, glucose, lipids, gestational weight gain (GWG), and so on. ${ }^{5-7}$ As a common adverse perinatal outcome, clinical doctors are concerned about decreasing and preventing the incidence of LGA.

Nonalcoholic fatty liver disease (NAFLD) is one of the most important causes of liver disease worldwide. ${ }^{8}$ A recent study reported the prevalence of NAFLD in the contemporary US population was a stagging $37.1 \%{ }^{9}$ As a multisystemic metabolic disorder, the close relationship of NAFLD and diabetes is confirmed. ${ }^{10}$ The independent association between NAFLD and adverse perinatal outcomes, such as postpartum hemorrhage and preterm birth, has also been reported. ${ }^{11}$ The prevalence of NAFLD in pregnancy has nearly tripled in the last decade, ${ }^{11}$ but the screening of NAFLD in asymptomatic individuals by ultrasonography or computed tomography is not cost-effective. Hepatic steatosis index (HSI) was a simple noninvasive index to detect NAFLD by BMI and liver enzymes, ${ }^{12}$ which can also be an assessment tool of other metabolic diseases and their complications. ${ }^{13,14}$ The liver plays an important role in glucose metabolism and liver enzymes are related to diabetes risk. A previous study reported that although there was not a clear threshold, ALT levels were positively associated with GDM risk. ${ }^{15,16}$ However, to our knowledge, HSI has not been investigated among pregnant women and its relationship to GDM and LGA is unclear. Thus, in this study, we analyzed the association between HSI and the subsequent risk of GDM and LGA in a prospective, double-center, observational cohort study in China. Previous studies also reported NAFLD liver fat score could predict NAFLD ${ }^{17}$ and triglyceride/highdensity lipoprotein cholesterol (TG/HDL-C) as well as triglyceride-glucose (TyG) index were both good predictors for GDM and LGA. ${ }^{18}$ Hence, we also did preliminary analyses to compare the predictive ability of these indices in this study.

\section{Materials and Methods}

This work was a sub-study of an ongoing prospective double-center observational cohort study aiming to find the biological markers of GDM in early pregnancy by urinary proteomics, which started in 2019 at Haidian District Maternal and Child Health Care Hospital and Chaoyang District Maternal and Child Health Care Hospital, Beijing, China (clinical trial number: NCT03246295).

The ethics committees of all participating centers approved the study protocol. Written informed consent was obtained from each participant and the study was performed in accordance with the Declaration of Helsinki as revised in 2013 .

\section{Study Participants}

At baseline, participants underwent a clinical investigation at the first prenatal visit in early pregnancy (6-12 weeks of gestation). For all participants in the present study, all available clinical and laboratory data were recorded and verified by two researchers at the same time.

Inclusion and exclusion criteria of participants were as follows. Inclusion criteria: (1) gestation at entry $<12$ weeks; (2) without diabetes mellitus before pregnancy; (3) acceptance of participation in the study, and signature of the consent form. Exclusion criteria: (1) non-singleton pregnancy; (2) fasting blood glucose $\geq 6.1 \mathrm{mmol} / \mathrm{L}$ at baseline; (3) severe acute or chronic diseases, such as severe liver and renal dysfunction, heart disease, autoimmune disease, and so on. On this basis, a total of 1128 pregnant women with clinical and laboratory data at 6-12 weeks of gestation and 75-g oral glucose tolerance test (OGTT) at 24-28 weeks of gestation were enrolled from August 2019 to November, 2020. Forty-six women without data on gestational weight gain were excluded. Thus 1082 pregnant women were included in the present study. Perinatal database of 826 women was collected from electronic medical records (EMRs).

\section{Measurements}

Participants were measured by body weight, height, systolic blood pressure (SBP) and diastolic blood pressure (DBP) at the first prenatal visit (6-12 weeks of gestation). Body weight and blood pressure were monitored during gestation. Blood samples collected from the peripheral 
vein of participants at the first prenatal visit were used to examine the parameters of blood routine, fasting blood glucose, insulin, C-peptide, total cholesterol (TC), TG, low-density lipoprotein cholesterol (LDL-C), HDL-C, and liver and renal function. Medical history, personal history and family history were recorded in EMR.

A 75-g OGTT was conducted for all participants at 24 28 weeks of gestation to screen GDM. GDM was diagnosed according to the International Association of the Diabetes and Pregnancy Study Groups criterion in 2010 . $^{1}$ LGA and small for gestational age (SGA) were defined as birth weight $>90$ th percentile and $<10$ th percentile for gestational age and sex, respectively. ${ }^{19}$

Body mass index (BMI) was determined by dividing body weight in kilograms by height in meters squared. HSI was calculated as $8 * \mathrm{ALT} / \mathrm{AST}+\mathrm{BMI}+2$ in women. ${ }^{12}$ NAFLD liver fat score was calculated according to its first publication. ${ }^{17}$ TG/HDL-C was TG to HDL-C ratio and TyG index was ln (fasting $\mathrm{TG}[\mathrm{mg} / \mathrm{dL}]^{*} \mathrm{FBG}[\mathrm{mg} /$ $\mathrm{dL}] / 2){ }^{18}$

\section{Statistical Analysis}

Statistical analyses were conducted using the Software Package for Social Science (SPSS version 24.0, SPSS, IBM Corporation, Armonk, NY, USA). Continuous variables were tested for normality of distribution. Variables with approximately normal distributions were presented as mean $\pm \mathrm{SD}$, and those with skewed distributions were presented as median and interquartile range (25th-75th percentile). Categorical variables were presented as percentage (number). Two-sample Student's $t$ test or Mann-Whitney test was used for continuous variables and chi-squared test or Fisher's exact test were used for categorical variables. HSI (quartile 1, $\leq 25.92$; quartile 2, 25.93-28.45; quartile 3, 28.46-31.78; quartile 4, $\geq 31.79$ ) were divided into quartiles according to the cutoff points of entire distribution for this study population. $P$-value for the linear trend of glucose concentration during OGTT, birth weight of infant, incidence of GDM and LGA based on HSI quartiles were calculated. Binary logistic regression analyses were performed to determine the odds ratio (ORs) and 95\% confidence intervals (CIs) of GDM and LGA according to HSI quartiles with adjustment for potential confounding factors. Receiver operating characteristic (ROC) curve analysis was used to evaluate the predictive ability of HSI, NAFLD liver fat score, TG/ HDL-C and TyG for GDM and LGA delivery. Z test was performed for comparison between ROC-AUC of HSI and other indices for GDM and LGA Infant (MedCalc, version 19.6.4). Statistical significance was inferred from twosided $P$-values $<0.05$.

\section{Results}

In the present study, the incidence of GDM was $22.09 \%$ (239/1082). Maternal and neonatal characteristics between women with and without GDM were shown in Table 1. Compared to women with normal glucose tolerance (NGT), women with GDM had significantly older age as well as higher pre-pregnancy body mass index (preBMI), percentage of family history of diabetes mellitus (FHDM), systolic blood pressure (SBP), FBG, insulin, C-peptide, ALT, TG, LDL-C, HSI, and other indices $(P<0.05)$, but lower gestational weight gain (BMI gain[B]) and HDL-C. Neonatal characteristics in women with recorded perinatal outcomes were also presented in Table 1. The overall incidence of LGA was $10.53 \%(87 / 826)$ and the incidence of LGA was higher in GDM group than in NGT group, but the difference was not significant $(13.9 \%$ vs $9.6 \%$, $P=0.107)$.

Values of HSI were divided into quartiles. Maternal glucose concentration at each time point during OGTT, infant birth weight, incidence of GDM, LGA, and SGA in each HSI quartile were presented in Table 2. Although the differences between two neighboring groups were slight, there were statistically significant increased linear trends between OGTT results, birth weight, incidence of GDM and increased HSI quartiles ( $P$ for linear trend $<0.05$ ). LGA in women with the third quartile of HSI was lower than in the second quartile, but $P$ for linear trend analysis implied the incidence of LGA increased with higher HSI in totality $(P=0.001)$. The incidence of SGA was gradually decreased from the lowest quartile of HSI to the highest quartile of HSI. Gravidity and parity had no significant difference among these groups (Supplementary Table 1). Since the originally proposed HSI cutoff points for diagnosing or excluding NAFLD were 30 and 36, the comparison of variables based on these points was shown in Supplementary Table 2. The incidence of GDM in women with HSI $>36$ reached up to $40 \%$.

The lowest quartile of HSI was used as reference and binary logistic regression analyses were used to examine the association between the quartiles of HSI and GDM as well as LGA risk (Table 3). Women in the upper two HSI quartiles had significantly higher GDM risk than those in the lowest quartile and after adjustment for confounding factors, the results were still significant (all $P<0.05$ ). 
Table I Maternal and Neonatal Characteristics Between Women with and without GDM

\begin{tabular}{|c|c|c|c|}
\hline Variables & GDM & NGT & $P$-value \\
\hline Maternal Characteristics & $n=239$ & $n=843$ & \\
\hline Age & $31.40 \pm 3.92$ & $30.08 \pm 3.93$ & $<0.001$ \\
\hline PreBMI $\left(\mathrm{kg} / \mathrm{m}^{2}\right)$ & $23.13 \pm 3.53$ & $21.68 \pm 2.81$ & $<0.001$ \\
\hline BMI gain $(A)\left(k g / m^{2}\right)$ & $2.5 \mathrm{I}(1.65-3.24)$ & $2.48(|.7|-3.3 \mid)$ & 0.802 \\
\hline BMI gain $(B)\left(k g / m^{2}\right)(n=734)$ & $3.91(3.05-5.14)$ & $4.96(3.86-5.88)$ & $<0.001$ \\
\hline FHDM & $43(18.0 \%)$ & 94 (II.2\%) & 0.005 \\
\hline Gravidity & & & 0.591 \\
\hline I & 119 (49.8\%) & 449 (53.3\%) & \\
\hline 2 & $68(28.5 \%)$ & 231 (27.4\%) & \\
\hline$\geq 3$ & $52(21.8 \%)$ & $163(19.3 \%)$ & \\
\hline Parity & & & 0.254 \\
\hline 0 & $159(66.5 \%)$ & $591(70.1 \%)$ & \\
\hline 1 & 75 (3I.4\%) & $244(28.9 \%)$ & \\
\hline$\geq 2$ & $5(2.1 \%)$ & $8(0.9 \%)$ & \\
\hline SBP $(\mathrm{mmHg})$ & $114.66 \pm 9.58$ & $112.56 \pm 10.50$ & 0.008 \\
\hline $\mathrm{DBP}(\mathrm{mmHg})$ & $71.21 \pm 8.52$ & $70.57 \pm 9.09$ & 0.352 \\
\hline FBG (mmol/L) & $4.8(4.4-5.2)$ & $4.5(4.1-4.9)$ & $<0.001$ \\
\hline Insulin $(\mu \mathrm{lU} / \mathrm{mL})(\mathrm{n}=958)$ & $8.10(4.90-10.90)$ & $6.10(4.30-8.40)$ & $<0.001$ \\
\hline C-peptide $(\mathrm{ng} / \mathrm{mL})(\mathrm{n}=958)$ & $0.95(0.69-1.31)$ & $0.79(0.60-1.04)$ & $<0.001$ \\
\hline ALT (U/L) & I5.28 (I I.00-20.80) & $12.56(10.00-17.70)$ & $<0.001$ \\
\hline AST (U/L) & $16.17(14.00-19.00)$ & $16.00(14.00-18.80)$ & 0.406 \\
\hline TBil $(\mu \mathrm{mol} / \mathrm{L})$ & $10.40(8.50-13.60)$ & $10.50(8.40-13.00)$ & 0.641 \\
\hline $\mathrm{TC}(\mathrm{mmol} / \mathrm{L})$ & $4.02(3.48-4.66)$ & $3.92(3.50-4.45)$ & 0.085 \\
\hline TG $(\mathrm{mmol} / \mathrm{L})$ & $0.95(0.72-1.32)$ & $0.83(0.63-1.10)$ & $<0.001$ \\
\hline HDL-C (mmol/L) & $1.41(1.20-1.61)$ & I.49 (I.29-I.7I) & $<0.001$ \\
\hline LDL-C (mmol/L) & $2.12(1.76-2.67)$ & $2.00(1.68-2.40)$ & 0.001 \\
\hline HSI & $30.67(27.20-35.10)$ & $27.98(25.70-30.82)$ & $<0.001$ \\
\hline NAFLD liver fat score $(n=958)$ & $2.02(1.35-2.57)$ & $1.53(1.22-1.96)$ & $<0.001$ \\
\hline TG/HDL-C & $0.68(0.48-0.92)$ & $0.55(0.4 \mathrm{I}-0.77)$ & $<0.001$ \\
\hline TyG & $8.20(7.93-8.51)$ & $8.00(7.68-8.29)$ & $<0.001$ \\
\hline Neonatal Characteristics & $n=173$ & $n=653$ & \\
\hline Gestational age (weeks) & $39.00(38.14-40.00)$ & $39.29(38.57-40.14)$ & $<0.001$ \\
\hline Birth weight (g) & $3310(3030-3600)$ & $3310(3000-3540)$ & 0.629 \\
\hline Sex (male) & 88 (50.9\%) & 347 (53.1\%) & 0.595 \\
\hline
\end{tabular}


Table I (Continued).

\begin{tabular}{|l|l|l|l|}
\hline Variables & GDM & NGT & $P$-value \\
\hline LGA & $24(13.9 \%)$ & $63(9.6 \%)$ & 0.107 \\
\hline SGA & $8(4.6 \%)$ & $42(6.4 \%)$ & 0.375 \\
\hline
\end{tabular}

Note: Data are presented as mean \pm SD or median (interquartile range) or number (\%).

Abbreviations: GDM, gestational diabetes mellitus; NGT, normal glucose tolerance; preBMI, pre-pregnancy body mass index; BMI gain (A): maternal BMI gain before OGTT; BMI gain (B): maternal BMI gain during gestation; FHDM, family history of diabetes mellitus; SBP, systolic blood pressure; DBP, diastolic blood pressure; FBG, fasting blood glucose; ALT, alanine aminotransferase; AST, aspartate aminotransferase; TBil, total bilirubin; TC, total cholesterol; TG, triglyceride; HDL-C, high-density lipoprotein cholesterol; LDL-C, low-density lipoprotein cholesterol; HSI, hepatic steatosis index; NAFLD, nonalcoholic fatty liver disease; TyG, triglyceride-glucose index; LGA, large for gestational age; SGA, small for gestational age.

Table 2 Glucose Concentration at Three Time Points During OGTT, Incidence of GDM, LGA and SGA According to HSI Quartiles

\begin{tabular}{|l|l|l|l|l|l|}
\hline Variables & Quartile I & Quartile 2 & Quartile 3 & Quartile 4 & P for Linear Trend \\
\hline $\mathrm{n}$ & 267 & 274 & 271 & 270 & - \\
\hline HSI range & $\leq 25.92$ & $25.93-28.45$ & $28.46-31.78$ & $\geq 31.79$ & - \\
\hline $\begin{array}{l}\text { OGTT (mmol/L) } \\
\text { FBG }\end{array}$ & $4.63(4.38-4.83)$ & $4.66(4.40-4.85)$ & $4.69(4.47-4.93)$ & $4.76(4.53-5.10)$ & $<0.00$ I \\
I-hBG & $7.36(6.26-8.30)$ & $7.18(6.38-8.37)$ & $7.71(6.68-8.78)$ & $8.12(7.04-9.27)$ & $<0.001$ \\
2-hBG & $6.14(5.50-7.14)$ & $6.31(5.65-7.05)$ & $6.60(5.86-7.50)$ & $6.92(6.10-7.82)$ & $<0.001$ \\
GDM & $38(14.2 \%)$ & $40(14.6 \%)$ & $68(25.1 \%)$ & $93(34.4 \%)$ & $<0.001$ \\
\hline n & 197 & 206 & 205 & 218 & - \\
\hline Birth weight (g) & $3240(2940-3460)$ & $3275(2990-3540)$ & $3350(3070-3580)$ & $3365(3070-3650)$ & 0.03 I \\
LGA & $8(4.1 \%)$ & $26(12.6 \%)$ & $19(9.3 \%)$ & $34(15.6 \%)$ & 0.00 I \\
SGA & $17(8.6 \%)$ & $14(6.8 \%)$ & $12(5.9 \%)$ & $7(3.2 \%)$ & 0.020 \\
\hline
\end{tabular}

Note: Data are presented as median (interquartile range) or number (\%).

Abbreviations: HSI, hepatic steatosis index; OGTT, oral glucose tolerance test; FBG, fasting blood glucose; GDM, gestational diabetes mellitus; LGA, large for gestational age; SGA, small for gestational age.

Compared to women in the lowest quartile, women in the upper quartile had higher risk of LGA delivery (all $P<0.05)$. After adjustment for confounding factors, including maternal age, gestational age, BMI gain in gestation, FHDM, FBG, TC, TG, HDL-C, LDL-C and even GDM, women in the second and highest HSI quartiles still had significantly higher LGA delivery risk (adjusted OR, 95\% CI: 3.441, 1.438-8.237; 3.444, 1.417-8.368, $P<0.05$, respectively).

The AUC values for detecting GDM and LGA infant by HSI, NAFLD liver fat score, TG/HDL-C and TyG index were shown in Table 4. The AUC of all indices for predicting GDM were statistically significant $(P<0.001$ for all, Table 4 and Figure 1). Although the differences of AUC between HSI and other indices were not statistically significant (Supplementary Table 3), the AUC of HSI was the highest among them $(0.646,95 \% \mathrm{CI}: 0.605-0.686$, $P<0.001)$. The AUC of HSI and other indices for detecting LGA infant were presented in Table 4 and Figure 2. In this cohort, HSI could be a predictor for LGA, whereas the predictive ability of other indices for LGA were not statistically significant $(0.600,95 \% \mathrm{CI}: \quad 0.541-0.660$, $P=0.002)$. The comparison between predictive ability of HSI and preBMI for GDM and LGA were in Supplementary Figure 1 and Supplementary Figure 2. Preliminary analysis on the predictive ability of all indices for SGA was also given in this study (Supplementary Figure 3).

\section{Discussion}

In this study, we examined the relationship of HSI in the first trimester and the incidence of GDM and LGA in Chinese pregnant women and compared them with other indices. Higher HSI levels were independently associated with higher subsequent risk of GDM and LGA. HSI was a relatively better simple index for predicting risk of GDM and LGA delivery. To the best of our knowledge, this is 
Table 3 Association Between HSI Quartiles and GDM and LGA

\begin{tabular}{|c|c|c|c|}
\hline Variables & Quartile 2 & Quartile 3 & Quartile 4 \\
\hline \multicolumn{4}{|l|}{ GDM } \\
\hline Crude & $1.030(0.637-1.665)$ & $2.019(1.301-3.133)$ & $3.166(2.069-4.845$ \\
\hline Multivariable-adjusted (A) & $0.970(0.579-1.575)$ & $1.882(1.207-2.935)$ & $2.915(1.889-4.498$ \\
\hline Multivariable-adjusted (B) & $0.847(0.515-1.394)$ & $1.623(1.027-2.566)$ & $2.100(1.322-3.336$ \\
\hline \multicolumn{4}{|l|}{ LGA } \\
\hline Crude & $3.413(1.506-7.735)$ & $2.4 \mid 3(1.03 \mid-5.649)$ & $4.365(1.968-9.681)$ \\
\hline Multivariable-adjusted (C) & $3.264(1.382-7.710)$ & $2.016(0.815-4.985)$ & $3.881(1.653-9.113$ \\
\hline Multivariable-adjusted (D) & 3.257 (1.370-7.742) & $\mathrm{I} .940(0.777-4.84 \mathrm{I})$ & $3.455(1.426-8.373$ \\
\hline Multivariable-adjusted (E) & 3.44 I (1.438-8.237) & $1.956(0.782-4.894)$ & $3.444(1.417-8.368$ \\
\hline
\end{tabular}

Notes: Data are presented as OR $(95 \% \mathrm{Cl})$. (A): Adjusted for age, BMI gain before OGTT and FHDM. (B):Adjusted for (A) + FBG, TC, TG, HDL-C and LDL-C. (C): Adjusted for age, gestational age, BMI gain in gestation and FHDM. (D): Adjusted for (C) + FBG, TC, TG, HDL-C and LDL-C. (E): Adjusted for (D) + GDM.

Abbreviations: GDM, gestational diabetes mellitus; LGA, large for gestational age; HSI, hepatic steatosis index; BMI, body mass index; FHDM, family history of diabetes mellitus; FBG, fasting blood glucose; TC, total cholesterol; TG, triglyceride; HDL-C, high-density lipoprotein cholesterol; LDL-C, low-density lipoprotein cholesterol.

Table 4 Area Under Curve (AUC) and Corresponding 95\% Confidence Interval (Cl) of the HSI and Other Indices for Detecting GDM Development and LGA Infant

\begin{tabular}{|l|c|c|c|c|c|c|}
\hline \multirow{2}{*}{ Variables } & \multicolumn{3}{|c|}{ GDM } & \multicolumn{3}{c|}{ LGA } \\
\cline { 2 - 7 } & AUC & $95 \%$ CI & P-value & AUC & $95 \%$ CI & P -value \\
\hline HSI & 0.646 & $0.605-0.686$ & $<0.001$ & 0.600 & $0.541-0.660$ & 0.002 \\
NAFLD liver fat score & 0.638 & $0.594-0.683$ & $<0.001$ & 0.555 & $0.485-0.625$ & 0.121 \\
TG/HDL-C & 0.606 & $0.566-0.646$ & $<0.001$ & 0.513 & $0.447-0.579$ & 0.701 \\
TyG & 0.636 & $0.596-0.675$ & $<0.001$ & 0.558 & $0.494-0.623$ & 0.074 \\
\hline
\end{tabular}

Abbreviations: GDM, gestational diabetes mellitus; LGA, large for gestational age; HSI, hepatic steatosis index; NAFLD, nonalcoholic fatty liver disease; TG/HDL-C, triglyceride/high-density lipoprotein cholesterol; TyG, triglyceride-glucose index.

the first study to evaluate the association of HSI in early pregnancy and the subsequent GDM and LGA risk.

The increasing prevalence of GDM in China is alarming over the past few decades. ${ }^{20}$ The prevalence of GDM varies in different cities and regions because of many factors, such as economics level, diet, education and so on. In this study, the incidence of GDM was much higher than average prevalence of GDM in China, but was similar to the previous investigation done in Beijing. ${ }^{21}$ The booming economy and good living conditions in Beijing, followed by the high prevalence of metabolic disorders, including GDM, indicating it is crucial to improve people's health consciousness. PreBMI was significantly higher in GDM group, which was consistent with previous studies. ${ }^{22}$ Gestational weight gain (GWG) was usually higher in women with GDM, ${ }^{22}$ but women who participated in this study underwent regular follow-up and professional guidance during pregnancy, and BMI increased during pregnancy in GDM group was significantly lower than those in the NGT group.

As a common public health problem, NAFLD has influenced nearly a third adult population worldwide. ${ }^{23,24}$
The pathogenesis of NAFLD is complicated, but it is acknowledged that dysfunctional hepatic lipid metabolism and insulin resistance are common features of NAFLD and contribute a lot to its progression. ${ }^{24,25}$ Metabolic syndrome (MetS) is a strong risk factor of NAFLD and NAFLD and MetS may be bidirectional. Among all the features of MetS, diabetes mellitus has the most obvious link to NAFLD, and $75 \%$ of individuals with T2DM may have NAFLD. ${ }^{26}$ Insulin resistance and dyslipidemia also provide appropriate conditions for the development of GDM and LGA, thus some researchers investigated the association of NAFLD and GDM as well as LGA. However, it seems the relationship between NAFLD and GDM is not completely similar to that between NAFLD and T2DM. There is bidirectional influence between NAFLD and T2DM, while NAFLD can increase the risk of GDM, but GDM may not have the same significant effect on the development of NAFLD. Souza et $\mathrm{al}^{27}$ found NAFLD in early pregnancy could predict dysglycemia in midpregnancy. Ciardullo et $\mathrm{al}^{28}$ investigated the relative impact of T2DM and previous GDM on the development 


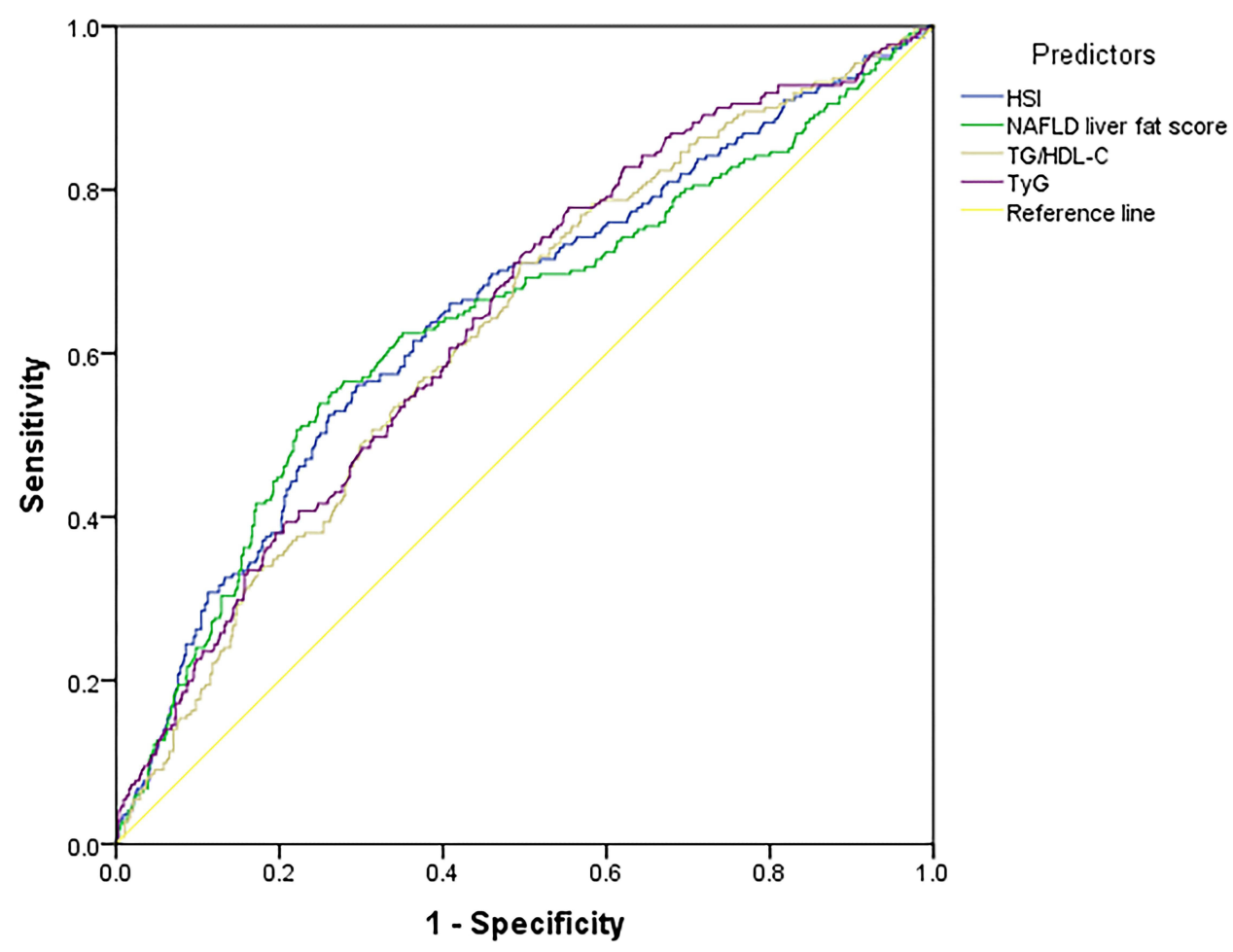

Figure I Receiver operating characteristic (ROC) curves of HSI and other indices used to predict GDM.

of significant liver fibrosis related to NAFLD by vibration controlled transient elastography and found current T2DM, rather than a previous history of GDM, was independently associated with steatosis and significant fibrosis. As for LGA, Lee et $\mathrm{al}^{29}$ reported NAFLD was a risk factor of LGA in a prospective cohort study including 623 pregnant women. NAFLD in most studies was diagnosed by ultrasound examination, which is not the prenatal routine examination. It is unclear whether the HSI, a simple screening tool for NAFLD, is related to GDM and LGA.

Our findings showed HSI was closely associated with glucose concentration during OGTT and the risk of GDM. The incidence of GDM in the highest HSI quartile was even up to $34.4 \%$. It is acknowledged that high preBMI is a strong risk factor of GDM. ${ }^{30}$ Higher liver enzymes, even in the normal range, have been noticed to be associated with increased diabetic risk, including GDM. ${ }^{31,32}$ ALT/ AST is a simple assessment tool of viral hepatitis, which has also been reported as a potential predictive index for metabolic diseases. ${ }^{33-35}$ Thus the association between HSI and GDM in this study was an expectation. This result further proved the independent relationship between NAFLD and GDM from another perspective by a simple index. NAFLD liver fat score is also a noninvasive index to diagnose NAFLD, but it is unclear whether it can predict GDM. TG/HDL-C and TyG have been proposed as simple clinical indicators of insulin resistance and previous studies found its potential clinical utility for predicting GDM. ${ }^{18,36}$ To further investigate the clinical value of HSI, we examined the predictive ability of HSI for GDM and compared it with the above indices. In this cohort study, HSI in first trimester appeared relatively higher predictive ability for GDM compared with other indices, although there was no statistical significance. As all clinical variables included in these indices are closely related to individual life habits and can be changed by diet, physical exercise or drugs, these results suggested that necessary interventions during pre-pregnancy in women with high risk of GDM might be an effective and economic way to decrease the incidence of GDM.

The results in this study showed the infant birth weight was increased with HSI increasing. Although the previous study related to the association of maternal HSI and infant birth weight and incidence of LGA is rare, our result was consistent with the effect of NAFLD on perinatal outcomes reported by Lee et al. ${ }^{29}$ The incidence of LGA were the lowest and highest in the first and fourth HSI quartiles, respectively. But it was lower in the third HSI 


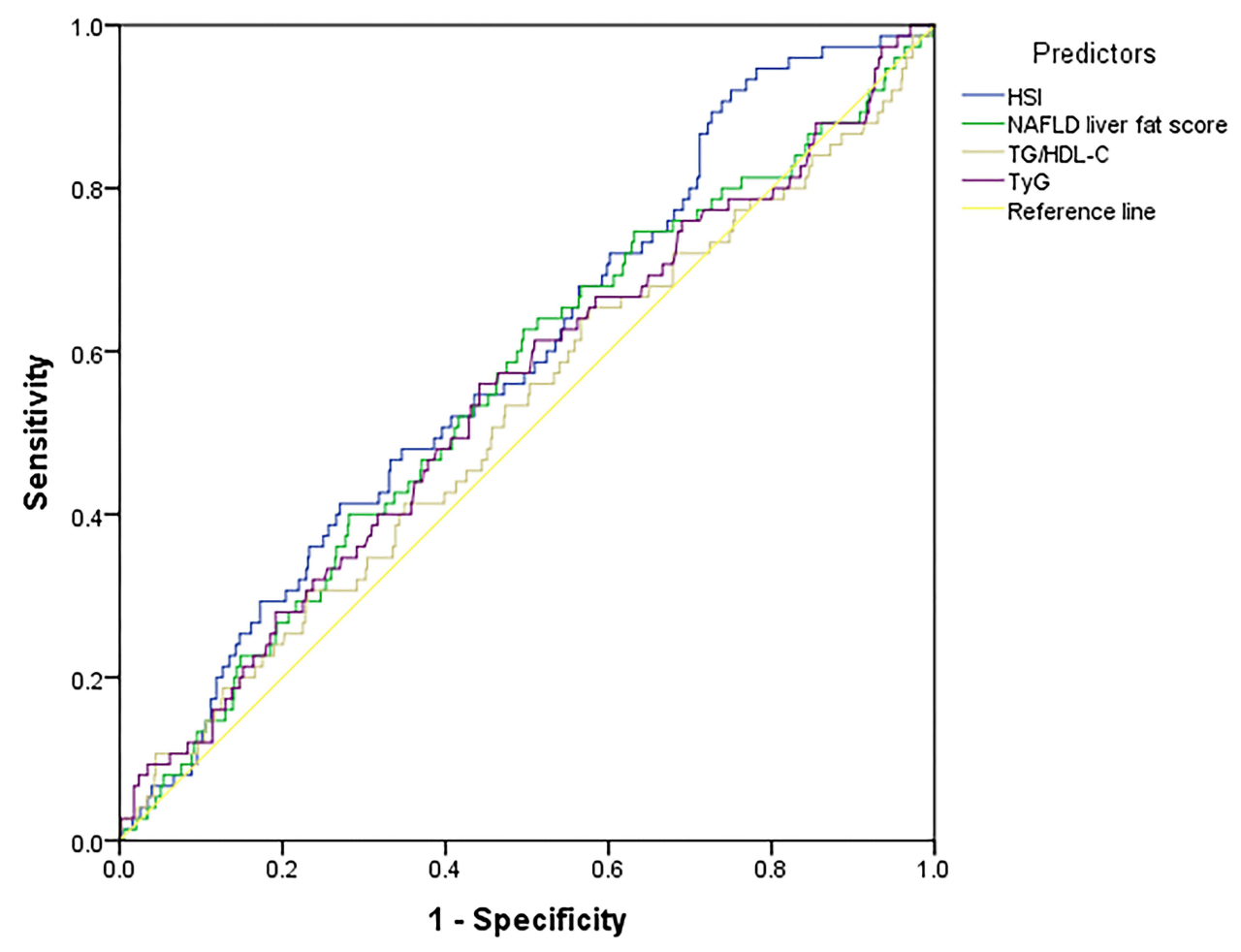

Figure 2 Receiver operating characteristic (ROC) curves of HSI and other indices used to predict LGA infant.

quartile than in the second quartile, and after adjustment for preBMI, GWG, FHDM, FBG, blood lipids, and GDM, the OR with $95 \% \mathrm{CI}$ for LGA in the second HSI quartile was not statistically significant. The most probable explanation might be that some potential confounding factors were not collected in this study, such as diet, physical exercise, blood lipids concentration during pregnancy, as well as percentage and dose of insulin therapy and glucose control condition in women with GDM. Previous studies reported maternal first-trimester TG/HDL-C and TyG were good indicators in predicting the risk of later LGA newborn. ${ }^{18,37}$ We also analyzed the predictive ability of HSI and NAFLD liver fat score, TG/HDL-C and TyG. To our surprise, HSI was an effective predictor for LGA while the other indices could not predict LGA risk in the present study. These results also indicated the complicated risk factors of LGA. The predictive ability of HSI for LGA should be further investigated in different cohorts.

The association between HSI and the subsequent risk of GDM was already obvious in the first trimester, and it also suggested increased risk of LGA had existed at early stage of pregnancy in women with high HSI. However, there are still some limitations that should be noted in this study. First, some potential confounding factors, as mentioned above, were not included in this study. Second, the samples of LGA infants were limited, which may lead to bias of results. Predicting GDM and LGA by simple and economical ways in the first trimester or even prepregnancy may allow for early interventions to decrease these risks. However, we have noticed the predictive ability of these indices, including TG/HDL-C and TyG, were all limited. It indicated that the development of GDM and LGA are complicated processes involving many factors. The predictive ability of existing indices might not be stable enough. But more study on related risk factors may contribute to build a potential prediction model for GDM and LGA in the future. Since the predictive ability of HSI was relatively better and more stable than other indices, being a more easily available index, the association between HSI and GDM as well as LGA deserves further investigation in larger population and different races.

\section{Conclusion}

In conclusion, in this study, higher HSI in early pregnancy was independently associated with higher risk of GDM and LGA. This preliminary cohort study determined that maternal first-trimester HSI could be useful in predicting subsequent risk of GDM and LGA delivery in Chinese pregnant women. As an easily available index in clinical 
practice, HSI can be of clinical utility in pregnant women, providing better clinical features of patients for clinical doctors and possibly guiding the therapeutic choice.

\section{Data Sharing Statement}

The data sets used and/or analyzed during the current study are available from the corresponding author on reasonable request.

\section{Ethics Approval}

The ethics committees of all participating centers approved the study protocol (Peking Union Medical College Hospital, Institute of Beijing Medical Science Chinese Academy of Medical Sciences, Haidian District Maternal and Child Health Care Hospital and Beijing Chaoyang District Maternal and Child Health Care Hospital) and the study was performed in accordance with the Declaration of Helsinki as revised in 2013.

\section{Consent}

All participants had given written consent to the inclusion of material pertaining to themselves and acknowledged they had been fully anonymized that they cannot be identified via the paper.

\section{Acknowledgments}

The authors thank all the participants in this study.

\section{Author Contributions}

All authors made a significant contribution to the work reported, whether that is in the conception, study design, execution, acquisition of data, analysis and interpretation, or in all these areas; took part in drafting, revising or critically reviewing the article; gave final approval of the version to be published; have agreed on the journal to which the article has been submitted; and agree to be accountable for all aspects of the work.

\section{Funding}

This study was supported by "13th Five-Year" National Science and Technology Major Project for New Drugs under Grant No. 2019ZX09734001 (to Weigang Zhao).

\section{Disclosure}

The authors report no conflicts of interest in this work.

\section{References}

1. Metzger BE, Gabbe SG, Persson B, et al. International association of diabetes and pregnancy study groups recommendations on the diagnosis and classification of hyperglycemia in pregnancy. Diabetes Care. 2010;33(3):676-682. doi:10.2337/dc09-1848

2. Billionnet C, Mitanchez D, Weill A, et al. Gestational diabetes and adverse perinatal outcomes from 716,152 births in France in 2012. Diabetologia. 2017;60(4):636-644. doi:10.1007/s00125-017-4206-6

3. Damm P, Houshmand-Oeregaard A, Kelstrup L, Lauenborg J, Mathiesen ER, Clausen TD. Gestational diabetes mellitus and long-term consequences for mother and offspring: a view from Denmark. Diabetologia. 2016;59(7):1396-1399. doi:10.1007/s0012 5-016-3985-5

4. Law GR, Alnaji A, Alrefaii L, et al. Suboptimal nocturnal glucose control is associated with large for gestational age in treated gestational diabetes mellitus. Diabetes Care. 2019;42(5):810-815. doi:10. 2337/dc18-2212

5. Goldstein RF, Abell SK, Ranasinha S, et al. Association of gestational weight gain with maternal and infant outcomes: a systematic review and meta-analysis. JAMA. 2017;317(21):2207-2225. doi:10. 1001/jama.2017.3635

6. HAPO Study Cooperative Research Group. The Hyperglycemia and Adverse Pregnancy Outcome (HAPO) Study. Int J Gynaecol Obstet. 2002;78(1):69-77. doi:10.1016/s0020-7292(02)00092-9

7. Jin WY, Lin SL, Hou RL, et al. Associations between maternal lipid profile and pregnancy complications and perinatal outcomes: a population-based study from China. BMC Pregnancy Childbirth. 2016;16(1):60. doi:10.1186/s12884-016-0852-9

8. Younossi Z, Anstee QM, Marietti M, et al. Global burden of NAFLD and NASH: trends, predictions, risk factors and prevention. Nat Rev Gastroenterol Hepatol. 2018;15(1):11-20. doi:10.1038/nrgastro.20 17.109

9. Ciardullo S, Perseghin G. Prevalence of NAFLD, MAFLD and associated advanced fibrosis in the contemporary United States population. Liver Int. 2021;41(6):1290-1293. doi:10.1111/liv.14828

10. Byrne CD, Targher G. NAFLD: a multisystem disease. J Hepatol. 2015;62(1 Suppl):S47-S64. doi:10.1016/j.jhep.2014.12.012

11. Sarkar M, Grab J, Dodge JL, et al. Non-alcoholic fatty liver disease in pregnancy is associated with adverse maternal and perinatal outcomes. J Hepatol. 2020;73(3):516-522. doi:10.1016/j.jhep.2020. 03.049

12. Lee JH, Kim D, Kim HJ, et al. Hepatic steatosis index: a simple screening tool reflecting nonalcoholic fatty liver disease. Dig Liver Dis. 2010;42(7):503-508. doi:10.1016/j.dld.2009.08.002

13. Sviklāne L, Olmane E, Dzērve Z, Kupčs K, Pīrāgs V, Sokolovska J. Fatty liver index and hepatic steatosis index for prediction of non-alcoholic fatty liver disease in type 1 diabetes. $J$ Gastroenterol Hepatol. 2018;33(1):270-276. doi:10.1111/jgh.13814

14. Ochiai H, Shirasawa T, Yoshimoto T, et al. Hepatic steatosis index and chronic kidney disease among middle-aged individuals: a large-scale study in Japan. Dis Markers. 2021;2021:9941834. doi:10.1155/2021/9941834

15. Leng J, Zhang C, Wang $\mathrm{P}$, et al. Plasma levels of alanine aminotransferase in the first trimester identify high risk Chinese women for gestational diabetes. Sci Rep. 2016;6(1):27291. doi:10.1038/srep 27291

16. Park JY, Kim WJ, Chung YH, et al. Association between pregravid liver enzyme levels and gestational diabetes in twin pregnancies: a secondary analysis of national cohort study. Sci Rep. 2021;11 (1):18695. doi:10.1038/s41598-021-98180-9

17. Kotronen A, Peltonen M, Hakkarainen A, et al. Prediction of non-alcoholic fatty liver disease and liver fat using metabolic and genetic factors. Gastroenterology. 2009;137(3):865-872. doi:10.10 53/j.gastro.2009.06.005 
18. Liu PJ, Liu Y, Ma L, et al. The predictive ability of two triglyceride-associated indices for gestational diabetes mellitus and large for gestational age infant among Chinese pregnancies: a preliminary cohort study. Diabetes Metab Syndr Obes. 2020;13:2025-2035. doi:10.2147/dmso.S251846

19. Dai L, Deng C, Li Y, et al. Birth weight reference percentiles for Chinese. PLoS One. 2014;9(8):e104779. doi:10.1371/journal. pone. 0104779

20. Juan J, Yang H. Prevalence, prevention, and lifestyle intervention of gestational diabetes mellitus in China. Int $J$ Environ Res Public Health. 2020;17(24):9517. doi:10.3390/ijerph17249517

21. Gao C, Sun X, Lu L, Liu F, Yuan J. Prevalence of gestational diabetes mellitus in mainland China: a systematic review and meta-analysis. $J$ Diabetes Investig. 2019;10(1):154-162. doi:10.1111/jdi.12854

22. Sun Y, Shen Z, Zhan Y, et al. Effects of pre-pregnancy body mass index and gestational weight gain on maternal and infant complications. BMC Pregnancy Childbirth. 2020;20(1):390. doi:10.1186/s12884-020-03071-y

23. Targher G, Byrne CD, Tilg H. NAFLD and increased risk of cardiovascular disease: clinical associations, pathophysiological mechanisms and pharmacological implications. Gut. 2020;69(9):1691-1705. doi:10.1136/gutjnl-2020-320622

24. Watt MJ, Miotto PM, De Nardo W, Montgomery MK. The liver as an endocrine organ-linking NAFLD and insulin resistance. Endocr Rev. 2019;40(5):1367-1393. doi:10.1210/er.2019-00034

25. Choudhury J, Sanyal AJ. Insulin resistance and the pathogenesis of nonalcoholic fatty liver disease. Clin Liver Dis. 2004;8(3):575-594. doi:10.1016/j.cld.2004.04.006

26. Friedman SL, Neuschwander-Tetri BA, Rinella M, Sanyal AJ. Mechanisms of NAFLD development and therapeutic strategies. Nat Med. 2018;24(7):908-922. doi:10.1038/s41591-018-0104-9

27. De Souza LR, Berger H, Retnakaran R, et al. Non-alcoholic fatty liver disease in early pregnancy predicts dysglycemia in mid-pregnancy: prospective study. Am J Gastroenterol. 2016;111 (5):665-670. doi:10.1038/ajg.2016.43

28. Ciardullo S, Bianconi E, Zerbini F, Perseghin G. Current type 2 diabetes, rather than previous gestational diabetes, is associated with liver disease in U.S. Women. Diabetes Res Clin Pract. 2021;177:108879. doi:10.1016/j.diabres.2021.108879
29. Lee SM, Kim BJ, Koo JN, et al. Nonalcoholic fatty liver disease is a risk factor for large-for-gestational-age birthweight. PLoS One. 2019;14(8):e0221400. doi:10.1371/journal.pone.0221400

30. Giannakou K, Evangelou E, Yiallouros P, et al. Risk factors for gestational diabetes: an umbrella review of meta-analyses of observational studies. PLoS One. 2019;14(4):e0215372. doi:10.1371/journal. pone. 0215372

31. Zhu Y, Hedderson MM, Quesenberry CP, Feng J, Ferrara A. Liver enzymes in early to mid-pregnancy, insulin resistance, and gestational diabetes risk: a longitudinal analysis. Front Endocrinol. 2018;9:581. doi:10.3389/fendo.2018.00581

32. Zhao W, Zhang L, Zhang G, et al. The association of plasma levels of liver enzymes and risk of gestational diabetes mellitus: a systematic review and dose-response meta-analysis of observational studies. Acta Diabetol. 2020;57(6):635-644. doi:10.1007/s00592-01901458-8

33. Yadav D, Choi E, Ahn SV, et al. Incremental predictive value of serum AST-to-ALT ratio for incident metabolic syndrome: the ARIRANG study. PLoS One. 2016;11(8):e0161304. doi:10.1371/ journal.pone.0161304

34. Warnakulasuriya LS, Samaranayake DL, Adikaram AVN, et al. Metabolic abnormalities in a cohort of overweight and obese children in an urban setting of Sri Lanka. Int $J$ Endocrinol. 2021;2021:9936889. doi:10.1155/2021/9936889

35. Kawamoto R, Kohara K, Kusunoki T, Tabara Y, Abe M, Miki T. Alanine aminotransferase/aspartate aminotransferase ratio is the best surrogate marker for insulin resistance in non-obese Japanese adults. Cardiovasc Diabetol. 2012;11(1):117. doi:10.1186/1475-2840-11-117

36. Kim JA, Kim J, Roh E, et al. Triglyceride and glucose index and the risk of gestational diabetes mellitus: a nationwide population-based cohort study. Diabetes Res Clin Pract. 2021;171:108533. doi:10.1016/j.diabres.2020.108533

37. Wang D, Xu S, Chen H, Zhong L, Wang Z. The associations between triglyceride to high-density lipoprotein cholesterol ratios and the risks of gestational diabetes mellitus and large-for-gestational-age infant. Clin Endocrinol. 2015;83(4):490-497. doi:10.1111/cen.12742

\section{Publish your work in this journal}

Diabetes, Metabolic Syndrome and Obesity: Targets and Therapy is an international, peer-reviewed open-access journal committed to the rapid publication of the latest laboratory and clinical findings in the fields of diabetes, metabolic syndrome and obesity research. Original research, review, case reports, hypothesis formation, expert opinion and commentaries are all considered for publication. The manuscript management system is completely online and includes a very quick and fair peer-review system, which is all easy to use. Visit http://www.dovepress.com/testimonials.php to read real quotes from published authors.

Submit your manuscript here: https://www.dovepress.com/diabetes-metabolic-syndrome-and-obesity-targets-and-therapy-journal 